\title{
The Nondifferentiable Solution for Local Fractional Tricomi Equation Arising in Fractal Transonic Flow by Local Fractional Variational Iteration Method
}

\author{
Ai-Min Yang, ${ }^{1,2}$ Yu-Zhu Zhang, ${ }^{2,3}$ and Xiao-Long Zhang ${ }^{1}$ \\ ${ }^{1}$ College of Science, Hebei United University, Tangshan 063009, China \\ ${ }^{2}$ College of Mechanical Engineering, Yanshan University, Qinhuangdao 066004, China \\ ${ }^{3}$ College of Metallurgy and Energy, Hebei United University, Tangshan 063009, China \\ Correspondence should be addressed to Yu-Zhu Zhang; zyz@heuu.edu.cn
}

Received 8 May 2014; Accepted 29 May 2014; Published 19 June 2014

Academic Editor: Xiao-Jun Yang

Copyright (C) 2014 Ai-Min Yang et al. This is an open access article distributed under the Creative Commons Attribution License, which permits unrestricted use, distribution, and reproduction in any medium, provided the original work is properly cited.

We present the nondifferentiable approximate solution for local fractional Tricomi equation arising in fractal transonic flow by local fractional variational iteration method. Some illustrative examples are shown and graphs are also given.

\section{Introduction}

In this paper, we study the local fractional Tricomi equation given as follows:

$$
\frac{y^{\alpha}}{\Gamma(1+\alpha)} \frac{\partial^{2 \alpha} u(x, y)}{\partial x^{2 \alpha}}+\frac{\partial^{2 \alpha} u(x, y)}{\partial y^{2 \alpha}}=0,
$$

where the quantity $u(x, y)$ is the nondifferentiable function and the operator is local fractional operator suggested as follows [1-3]:

$$
\frac{\partial^{\alpha} u(x, t)}{\partial t^{\alpha}}=\frac{\Delta^{\alpha}\left(u(x, t)-u\left(x, t_{0}\right)\right)}{\left(t-t_{0}\right)^{\alpha}},
$$

where

$$
\begin{aligned}
& \Delta^{\alpha}\left(u(x, t)-u\left(x, t_{0}\right)\right) \\
& \quad \cong \Gamma(1+\alpha)\left[u(x, t)-u\left(x, t_{0}\right)\right] .
\end{aligned}
$$

Local fractional derivative was applied to deal with nondifferentiable phenomena arising in mathematical physics [49]. When the fractal dimension $\alpha$ is equal to 1 , we obtain the following differential equation:

$$
y \frac{\partial^{2} u(x, y)}{\partial x^{2}}+\frac{\partial^{2} u(x, y)}{\partial y^{2}}=0
$$

which is structured by Tricomi [10]. The Tricomi equation was used to describe the transonic flow [10-22].
Local fractional variational iteration method first structured in [4] was an efficient tool to solve the local fractional differential equations, such as the fractal heat equation [4], the damped and dissipative wave equation in fractal strings [5], the wave equation on Cantor sets [6], the local fractional Poisson equation [7], the local fractional Laplace equation [8], and the local fractional Helmholtz equation [9]. The aim of this paper is to use the local fractional variational iteration method to deal with the local fractional Tricomi equation which arises in fractal transonic flow. The paper is organized as follows. In Section 2, the local fractional calculus theory is introduced. In Section 3, the local fractional variational iteration method is presented. In Section 4, the local fractional Tricomi equation is discussed. Finally, the conclusions are presented in Section 5.

\section{Local Fractional Calculus Theory}

In this section, we present the local fractional calculus theory, which is used in the present paper.

Definition 1 (see $[1,2])$. One has the function $f(x) \in C_{\alpha}(a, b)$, if

$$
\left|f(x)-f\left(x_{0}\right)\right|<\varepsilon^{\alpha}, \quad 0<\alpha \leq 1,
$$

is valid, where $\left|x-x_{0}\right|<\delta$, for $\varepsilon, \delta>0$ and $\varepsilon \in R$. 
Definition 2 (see $[1,4-9]$ ). Let $f(x)$ satisfy condition (5). The local fractional integral of $f(x)$ of order $\alpha$ in the interval $[a, b]$ is defined through

$$
\begin{aligned}
{ }_{a} I_{b}{ }^{(\alpha)} f(x) & =\frac{1}{\Gamma(1+\alpha)} \int_{a}^{b} f(t)(d t)^{\alpha} \\
& =\frac{1}{\Gamma(1+\alpha)} \lim _{\Delta t \rightarrow 0} \sum_{j=0}^{j=N-1} f\left(t_{j}\right)\left(\Delta t_{j}\right)^{\alpha}
\end{aligned}
$$

where the partitions of the interval $[a, b]$ are $\left(t_{j}, t_{j+1}\right), j=$ $0, \ldots, N-1, t_{0}=a$, and $t_{N}=b$ with $\Delta t_{j}=t_{j+1}-t_{j}$ and $\Delta t=\max \left\{\Delta t_{0}, \Delta t_{1}, \Delta t_{j}, \ldots\right\}$.

Definition 3 (see $[1,4-9]$ ). Let $f(x)$ satisfy condition (5). The inverse formula of (6) is given as follows:

$$
\frac{d^{\alpha} f\left(x_{0}\right)}{d x^{\alpha}}=D_{x}{ }^{(\alpha)} f\left(x_{0}\right)=\frac{\Delta^{\alpha}\left(f(x)-f\left(x_{0}\right)\right)}{\left(x-x_{0}\right)^{\alpha}},
$$

where

$$
\Delta^{\alpha}\left(f(x)-f\left(x_{0}\right)\right) \cong \Gamma(1+\alpha)\left[f(x)-f\left(x_{0}\right)\right] .
$$

The formulas of local fractional derivative and integral used in the paper are presented as follows $[1,6,7]$ :

$$
\begin{gathered}
\frac{d^{\alpha}}{d x^{\alpha}} \frac{x^{n \alpha}}{\Gamma(1+n \alpha)}=\frac{x^{(n-1) \alpha}}{\Gamma(1+(n-1) \alpha)}, \quad n \in N, \\
D_{x}{ }^{(\alpha)} a=0, \\
D_{x}{ }^{(\alpha)} a g(x)=a D_{x}{ }^{(\alpha)} g(x), \\
D_{x}{ }^{(\alpha)}\left[D_{x}{ }^{(\alpha)} f(x)\right]=D_{x}{ }^{(2 \alpha)} f(x), \\
{ }_{0} I_{t}^{(\alpha)}\left(\frac{{ }^{(t-s)}{ }^{\alpha} t^{n \alpha}}{\Gamma(1+\alpha) \Gamma(1+n \alpha)}\right)=\frac{t^{(n+2) \alpha}}{\Gamma(1+(n+2) \alpha)}, \\
{ }_{0} I_{x}{ }^{(\alpha)} \frac{x^{(n-1) \alpha}}{\Gamma(1+(n-1) \alpha)}=\frac{x^{n \alpha}}{\Gamma(1+n \alpha)}, \quad n \in N,
\end{gathered}
$$

where $g(x)$ is a local fractional continuous function, $a$ is a constant, and $N$ is a set of positive integers.

\section{Local Fractional Variational Iteration Method}

In this section, we introduce the local fractional variational iteration method. In order to show it, we consider the following local fractional operator equation:

$$
L_{\alpha}^{(2)} u+R_{\alpha} u=0
$$

where $L_{\alpha}^{(2)}$ denotes the linear local fractional differential operator and $R_{\alpha}$ denotes the linear local fractional differential operators of order less than $L_{\alpha}^{(2)}$.
According to the local fractional variational iteration method [4-9], we have a local fractional correction functional. Consider

$$
\begin{aligned}
u_{n+1}(x)= & u_{n}(x)+\frac{1}{\Gamma(1+\alpha)} \\
& \times \int_{0}^{x} \frac{\lambda^{\alpha}}{\Gamma(1+\alpha)}\left\{L_{\alpha}^{(2)} u_{n}(s)+R_{\alpha} \tilde{u}_{n}(s)\right\}(d s)^{\alpha}
\end{aligned}
$$

so that the local fractional variational iteration algorithm can be written as follows:

$$
\begin{aligned}
u_{n+1}(x)= & u_{n}(x)+\frac{1}{\Gamma(1+\alpha)} \\
& \times \int_{0}^{x} \frac{\lambda^{\alpha}}{\Gamma(1+\alpha)}\left\{L_{\alpha}^{(2)} u_{n}(s)+R_{\alpha} u_{n}(s)\right\}(d s)^{\alpha},
\end{aligned}
$$

where $\tilde{u}_{n}$ is as a restricted local fractional variation [1]; that is, $\delta^{\alpha} \widetilde{u}_{n}=0$.

Therefore, for $n \in N$, we give

$$
\begin{aligned}
\delta^{\alpha} u_{n+1}= & {\left[1-\left.\left(\frac{\lambda^{\alpha}}{\Gamma(1+\alpha)}\right)^{(\alpha)}\right|_{s=x}\right] \delta^{\alpha} u_{n} } \\
& +\left.\frac{\lambda^{\alpha}}{\Gamma(1+\alpha)}\right|_{s=x} \delta^{\alpha} \frac{\partial^{\alpha} u_{n}}{\partial x^{\alpha}} \\
& +{ }_{0} I_{x}^{(\alpha)}\left\{\left.\left(\frac{\lambda^{\alpha}}{\Gamma(1+\alpha)}\right)^{(2 \alpha)}\right|_{s=x} \delta^{\alpha} u_{n}\right\} .
\end{aligned}
$$

From (13), we obtain the stationary condition as follows:

$$
\begin{gathered}
1-\left.\left(\frac{\lambda^{\alpha}}{\Gamma(1+\alpha)}\right)^{(\alpha)}\right|_{s=x}=0,\left.\quad \frac{\lambda^{\alpha}}{\Gamma(1+\alpha)}\right|_{s=x}=0 \\
\left.\left(\frac{\lambda^{\alpha}}{\Gamma(1+\alpha)}\right)^{(2 \alpha)}\right|_{s=x}=0
\end{gathered}
$$

Then, the fractal Lagrange multiplier is

$$
\frac{\lambda^{\alpha}}{\Gamma(1+\alpha)}=\frac{(s-x)^{\alpha}}{\Gamma(1+\alpha)} \text {. }
$$

Making use of (12) and (15), we have the local fractional interaction formula as follows:

$$
\begin{aligned}
u_{n+1}(x)= & u_{n}(x)+\frac{1}{\Gamma(1+\alpha)} \\
& \times \int_{0}^{x} \frac{(s-x)^{\alpha}}{\Gamma(1+\alpha)}\left\{L_{\alpha}^{(2)} u_{n}(s)+R_{\alpha} u_{n}(s)\right\}(d s)^{\alpha} .
\end{aligned}
$$

Therefore, from (16), we get the solution given by

$$
u(x)=\lim _{n \rightarrow \infty} u_{n}(x) \text {. }
$$




\section{The Initial-Boundary Value Problems for Local Fractional Tricomi Equation}

In this section, we discuss the initial-boundary value problems for local fractional Tricomi equation.

Example 1. Let us consider the initial-boundary value conditions for the local fractional Tricomi equation as follows:

$$
\begin{gathered}
u(0, y)=0, \\
u(l, y)=0, \\
u(x, 0)=\frac{x^{2 \alpha}}{\Gamma(1+2 \alpha)}, \\
\frac{\partial^{\alpha} u(x, 0)}{\partial x^{\alpha}}=0 .
\end{gathered}
$$

From (18), (20), and (21), we have

$$
\begin{aligned}
u_{n+1}(x, y) & \\
= & u_{n}(x, y)+{ }_{0} I_{y}{ }^{(\alpha)} \\
\times & \left\{\frac{(s-y)^{\alpha}}{\Gamma(1+\alpha)}\right. \\
& \left.\times\left(\frac{s^{\alpha}}{\Gamma(1+\alpha)} \frac{\partial^{2 \alpha} u_{n}(x, s)}{\partial x^{2 \alpha}}+\frac{\partial^{2 \alpha} u_{n}(x, s)}{\partial y^{2 \alpha}}\right)\right\},
\end{aligned}
$$

where the initial value is given by

$$
u_{0}(x, y)=\frac{x^{2 \alpha}}{\Gamma(1+2 \alpha)}
$$

From (22), we present the first approximate formula as follows:

$$
\begin{aligned}
u_{1}(x, y) & \\
= & u_{0}(x, y)+{ }_{0} I_{y}{ }^{(\alpha)} \\
& \times\left\{\frac{(s-y)^{\alpha}}{\Gamma(1+\alpha)}\right. \\
& \left.\quad \times\left(\frac{s^{\alpha}}{\Gamma(1+\alpha)} \frac{\partial^{2 \alpha} u_{0}(x, s)}{\partial x^{2 \alpha}}+\frac{\partial^{2 \alpha} u_{0}(x, s)}{\partial y^{2 \alpha}}\right)\right\} \\
= & \frac{x^{2 \alpha}}{\Gamma(1+2 \alpha)}+\frac{y^{3 \alpha}}{\Gamma(1+3 \alpha)}
\end{aligned}
$$

and its graph is shown in Figure 1.

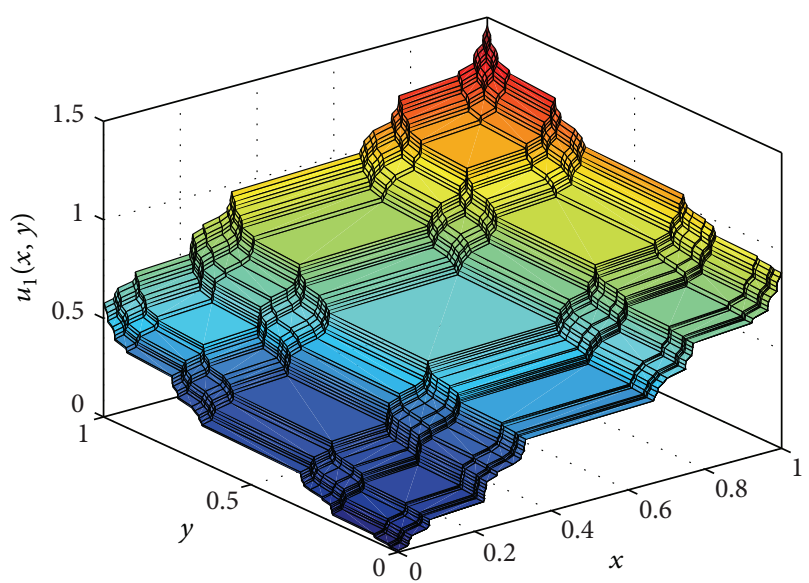

FIgURE 1: The plot of $u_{1}(x, y)$ with the parameter $\alpha=\ln 2 / \ln 3$.

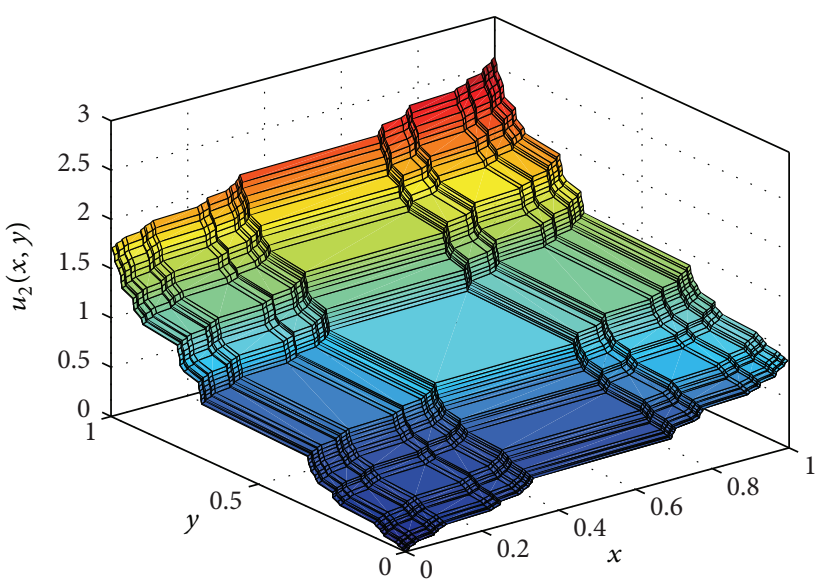

FIGURE 2: The plot of $u_{2}(x, y)$ with the parameter $\alpha=\ln 2 / \ln 3$.

The second approximate term is

$$
\begin{aligned}
u_{2}(x, y) & \\
= & u_{1}(x, y)+{ }_{0} I_{y}{ }^{(\alpha)} \\
& \times\left\{\frac{(s-y)^{\alpha}}{\Gamma(1+\alpha)}\right. \\
& \left.\quad \times\left(\frac{s^{\alpha}}{\Gamma(1+\alpha)} \frac{\partial^{2 \alpha} u_{1}(x, s)}{\partial x^{2 \alpha}}+\frac{\partial^{2 \alpha} u_{1}(x, s)}{\partial y^{2 \alpha}}\right)\right\} \\
= & \frac{x^{2 \alpha}}{\Gamma(1+2 \alpha)}+\frac{y^{3 \alpha}}{\Gamma(1+3 \alpha)} \\
& +{ }_{0} I_{y}{ }^{(\alpha)}\left\{\frac{(s-y)^{\alpha}}{\Gamma(1+\alpha)}\left(\frac{s^{\alpha}}{\Gamma(1+\alpha)}+\frac{s^{\alpha}}{\Gamma(1+\alpha)}\right)\right\} \\
= & \frac{x^{2 \alpha}}{\Gamma(1+2 \alpha)}+\frac{3 y^{3 \alpha}}{\Gamma(1+3 \alpha)}
\end{aligned}
$$

and its graph is given in Figure 2. 


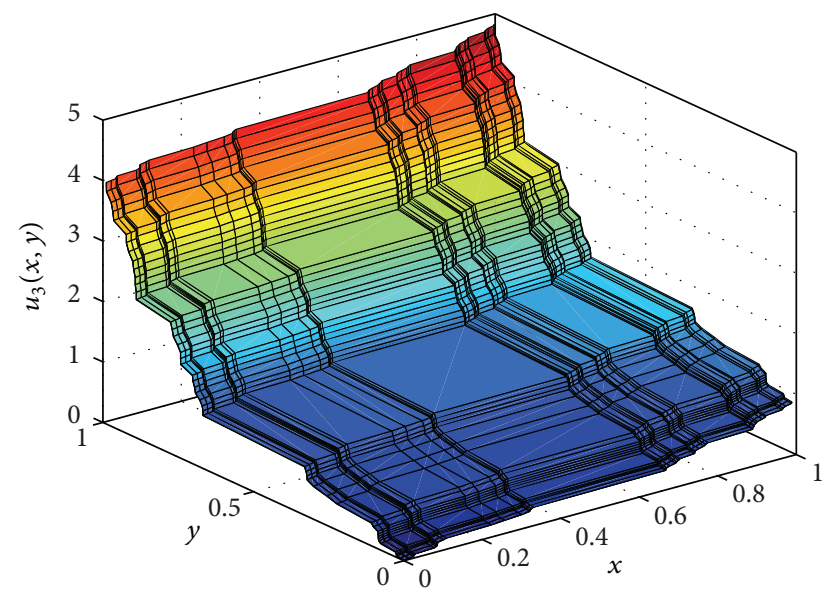

Figure 3: The plot of $u_{3}(x, y)$ with the parameter $\alpha=\ln 2 / \ln 3$.

The third approximation is presented as follows:

$$
\begin{aligned}
u_{3}(x, y) & \\
= & u_{2}(x, y)+{ }_{0} I_{y}{ }^{(\alpha)} \\
& \times\left\{\frac{(s-y)^{\alpha}}{\Gamma(1+\alpha)}\right. \\
= & \frac{x^{2 \alpha}}{\Gamma(1+2 \alpha)}+\frac{3 y^{3 \alpha}}{\Gamma(1+3 \alpha)} \\
& +{ }_{0} I_{y}{ }^{(\alpha)}\left\{\frac{(s-y)^{\alpha}}{\Gamma(1+\alpha)}\left(\frac{\partial^{2 \alpha} u_{2}(x, s)}{\partial x^{2 \alpha}}+\frac{\partial^{2 \alpha} u_{2}(x, s)}{\partial y^{2 \alpha}}\right)\right\} \\
= & \left.\left.\frac{x^{2 \alpha}}{\Gamma(1+2 \alpha)}+\frac{3 s^{\alpha}}{\Gamma(1+\alpha)}\right)\right\}
\end{aligned}
$$

and its graph is illustrated in Figure 3.

The fourth approximation reads as follows:

$$
\begin{aligned}
& u_{4}(x, y) \\
& =u_{3}(x, y)+{ }_{0} I_{y}{ }^{(\alpha)} \\
& \quad \times\left\{\frac{(s-y)^{\alpha}}{\Gamma(1+\alpha)}\right. \\
& \left.\quad \times\left(s^{\alpha} \frac{\partial^{2 \alpha} u_{3}(x, s)}{\partial x^{2 \alpha}}+\frac{\partial^{2 \alpha} u_{3}(x, s)}{\partial y^{2 \alpha}}\right)\right\}
\end{aligned}
$$

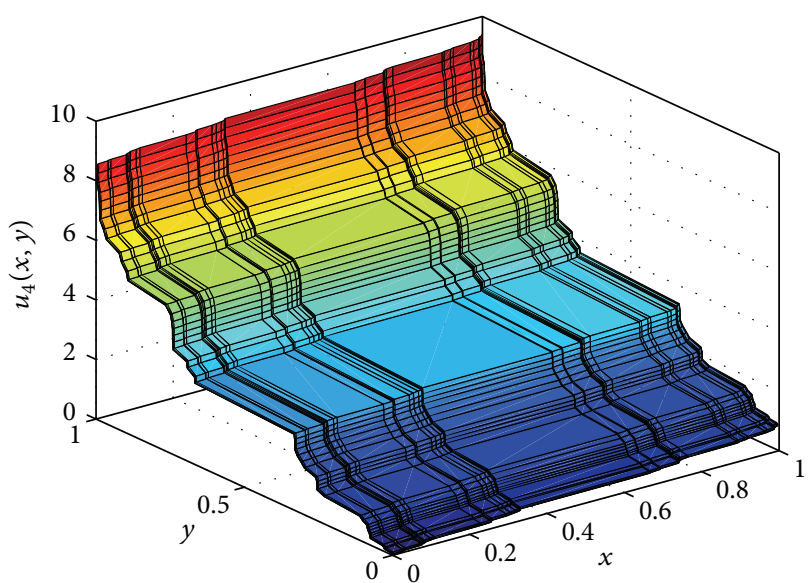

FIGURE 4: The plot of $u_{4}(x, y)$ with the parameter $\alpha=\ln 2 / \ln 3$.

$$
\begin{aligned}
= & \frac{x^{2 \alpha}}{\Gamma(1+2 \alpha)}+\frac{7 y^{3 \alpha}}{\Gamma(1+3 \alpha)} \\
& +{ }_{0} I_{y}{ }^{(\alpha)}\left\{\frac{(s-y)^{\alpha}}{\Gamma(1+\alpha)}\left(\frac{s^{\alpha}}{\Gamma(1+\alpha)}+\frac{7 s^{\alpha}}{\Gamma(1+\alpha)}\right)\right\} \\
= & \frac{x^{2 \alpha}}{\Gamma(1+2 \alpha)}+\frac{15 y^{3 \alpha}}{\Gamma(1+3 \alpha)}
\end{aligned}
$$

and its graph is presented in Figure 4.

The fifth approximation is as follows:

$$
\begin{aligned}
u_{5}(x, y) & \\
= & u_{4}(x, y)+{ }_{0} I_{y}{ }^{(\alpha)} \\
& \times\left\{\frac{(s-y)^{\alpha}}{\Gamma(1+\alpha)}\right. \\
& \left.\times\left(\frac{s^{\alpha}}{\Gamma(1+\alpha)} \frac{\partial^{2 \alpha} u_{4}(x, s)}{\partial x^{2 \alpha}}+\frac{\partial^{2 \alpha} u_{4}(x, s)}{\partial y^{2 \alpha}}\right)\right\} \\
& +x_{0}^{2 \alpha} I_{y}^{(\alpha)}\left\{\frac{(s-y)^{\alpha}}{\Gamma(1+\alpha)}\left(\frac{15 y^{3 \alpha}}{\Gamma(1+\alpha)}+\frac{15 s^{\alpha}}{\Gamma(1+\alpha)}\right)\right\} \\
= & \frac{x^{2 \alpha}}{\Gamma(1+2 \alpha)}+\frac{31 y^{3 \alpha}}{\Gamma(1+3 \alpha)}
\end{aligned}
$$

and its graph is shown in Figure 5.

After successive iterative processes, we obtain the nondifferentiable series solution as follows:

$$
\begin{aligned}
u(x, y) & =\lim _{i \rightarrow \infty} u_{n}(x, y) \\
& =\lim _{n \rightarrow \infty}\left\{\frac{x^{2 \alpha}}{\Gamma(1+2 \alpha)}+\left(2^{n}-1\right) \frac{y^{3 \alpha}}{\Gamma(1+3 \alpha)}\right\},
\end{aligned}
$$




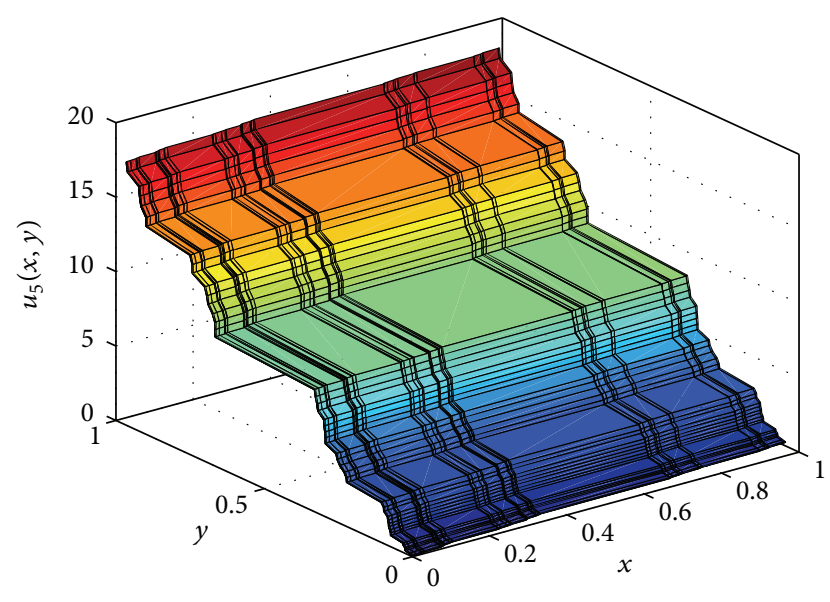

FIgURE 5: The plot of $u_{5}(x, y)$ with the parameter $\alpha=\ln 2 / \ln 3$.

which is the local fractional divergent series. Therefore, we can obtain the approximate solution.

Example 2. The initial-boundary value conditions for the local fractional Tricomi equation are presented as follows:

$$
\begin{gathered}
u(0, y)=0, \\
u(l, y)=0, \\
u(x, 0)=\frac{x^{\alpha}}{\Gamma(1+\alpha)}, \\
\frac{\partial^{\alpha} u(x, 0)}{\partial x^{\alpha}}=\frac{x^{\alpha}}{\Gamma(1+\alpha)} .
\end{gathered}
$$

In view of (16), (32), and (33), we obtain the local fractional iterative formula as follows:

$$
\begin{aligned}
u_{n+1}(x, y) & \\
= & u_{n}(x, y)+{ }_{0} I_{y}{ }^{(\alpha)} \\
\times & \left\{\frac{(s-y)^{\alpha}}{\Gamma(1+\alpha)}\right. \\
& \left.\times\left(\frac{s^{\alpha}}{\Gamma(1+\alpha)} \frac{\partial^{2 \alpha} u_{n}(x, s)}{\partial x^{2 \alpha}}+\frac{\partial^{2 \alpha} u_{n}(x, s)}{\partial y^{2 \alpha}}\right)\right\},
\end{aligned}
$$

with the initial value suggested as follows:

$$
u_{0}(x, y)=\frac{x^{\alpha}}{\Gamma(1+\alpha)}+\frac{x^{\alpha}}{\Gamma(1+\alpha)} \frac{y^{\alpha}}{\Gamma(1+\alpha)} .
$$

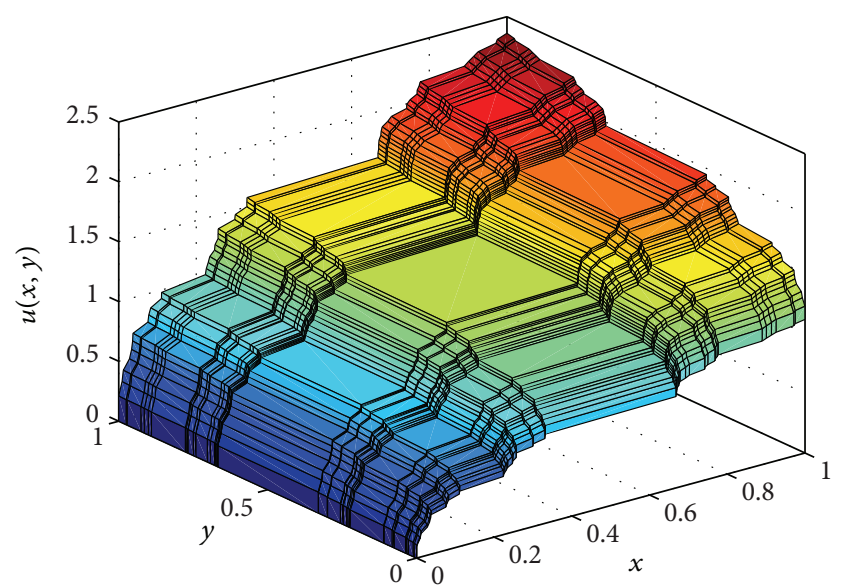

Figure 6: The plot of $u(x, y)$ with the parameter $\alpha=\ln 2 / \ln 3$.

From (34) and (35), we give the first approximation as follows:

$$
\begin{aligned}
u_{1}(x, y) & \\
= & u_{0}(x, y)+{ }_{0} I_{y}{ }^{(\alpha)} \\
& \times\left\{\frac{(s-y)^{\alpha}}{\Gamma(1+\alpha)}\right. \\
& \left.\quad \times\left(\frac{s^{\alpha}}{\Gamma(1+\alpha)} \frac{\partial^{2 \alpha} u_{0}(x, s)}{\partial x^{2 \alpha}}+\frac{\partial^{2 \alpha} u_{0}(x, s)}{\partial y^{2 \alpha}}\right)\right\} \\
= & \frac{x^{\alpha}}{\Gamma(1+\alpha)}+\frac{x^{\alpha}}{\Gamma(1+\alpha)} \frac{y^{\alpha}}{\Gamma(1+\alpha)} .
\end{aligned}
$$

Hence, from (36), we arrive at the following results:

$$
u_{0}(x, y)=u_{1}(x, y)=u_{2}(x, y)=\cdots=u_{n}(x, y) .
$$

Therefore, we get the exact solution with nondifferential term as follows:

$$
\begin{aligned}
u(x, y) & =\lim _{i \rightarrow \infty} u_{n}(x, y) \\
& =\lim _{n \rightarrow \infty}\left\{\frac{x^{\alpha}}{\Gamma(1+\alpha)}+\frac{x^{\alpha}}{\Gamma(1+\alpha)} \frac{y^{\alpha}}{\Gamma(1+\alpha)}\right\} \\
& =\frac{x^{\alpha}}{\Gamma(1+\alpha)}+\frac{x^{\alpha}}{\Gamma(1+\alpha)} \frac{y^{\alpha}}{\Gamma(1+\alpha)}
\end{aligned}
$$

and its graph is shown in Figure 6.

\section{Conclusions}

The initial-boundary value problems for local fractional Tricomi equation arising in fractal transonic flow based upon the local fractional derivatives are discussed. The solutions with nondifferentiable terms are obtained by using the local fractional variational iteration method and their graphs are also given to show the implement of the present method. 


\section{Conflict of Interests}

The authors declare that they have no competing interests in this paper.

\section{Acknowledgments}

This work was supported by National Scientific and Technological Support Projects (no. 2012BAE09B00), the National Natural Science Foundation of China (no. 51274270), and the National Natural Science Foundation of Hebei Province (no. E2013209215).

\section{References}

[1] X.-J. Yang, Advanced Local Fractional Calculus and Its Applications, World Science, New York, NY, USA, 2012.

[2] A. M. Yang, Y. Z. Zhang, and Y. Long, "The Yang-Fourier transforms to heat-conduction in a semi-infinite fractal bar," Thermal Science, vol. 17, no. 3, pp. 707-713, 2013.

[3] J. He, "Exp-function method for fractional differential equations," International Journal of Nonlinear Sciences and Numerical Simulation, vol. 14, no. 6, pp. 363-366, 2013.

[4] X.-J. Yang and D. Baleanu, "Fractal heat conduction problem solved by local fractional variation iteration method," Thermal Science, vol. 17, no. 2, pp. 625-628, 2013.

[5] W. Su, D. Baleanu, and X. a. . Yang, "Damped wave equation and dissipative wave equation in fractal strings within the local fractional variational iteration method," Fixed Point Theory and Applications, vol. 2013, article 89, 2013.

[6] D. Baleanu, J. A. T. Machado, C. Cattani, M. C. Baleanu, and $\mathrm{X}$. Yang, "Local fractional variational iteration and decomposition methods for wave equation on Cantor sets within local fractional operators," Abstract and Applied Analysis, vol. 2014, Article ID 535048, 6 pages, 2014.

[7] L. Chen, Y. Zhao, H. Jafari, J. A. Tenreiro Machado, and X. J. Yang, "Local fractional variational iteration method for local fractional Poisson equations in two independent variables," Abstract and Applied Analysis, vol. 2014, Article ID 484323, 7 pages, 2014.

[8] X. J. Yang, Y. J. Yang, and D. Baleanu, "A local fractional variational iteration method for Laplace equation within local fractional operators," Abstract and Applied Analysis, vol. 2013, Article ID 202650, 6 pages, 2013.

[9] A. M. Yang, Z. Chen, H. M. Srivastava, and X. J. Yang, "Application of the local fractional series expansion method and the variational iteration method to the Helmholtz equation involving local fractional derivative operators," Abstract and Applied Analysis, vol. 2013, Article ID 259125, 6 pages, 2013.

[10] F. Tricomi, "On second-order linear partial differential equations of mixed type," Moscow, Russia, 1947.

[11] A. Weinstein, "The singular solutions and the Cauchy problem for generalized Tricomi equations," Communications on Pure and Applied Mathematics, vol. 7, no. 1, pp. 105-116, 1954.

[12] T. Katsanis, "Numerical solution of Tricomi equation using theory of symmetric positive differential equations," SIAM Journal on Numerical Analysis, vol. 6, no. 2, pp. 236-253, 1969.

[13] C. S. Morawetz, "The Dirichlet problem for the Tricomi equation," Communications on Pure and Applied Mathematics, vol. 23, no. 4, pp. 587-601, 1970.
[14] K. R. Payne, "Interior regularity of the Dirichlet problem for the Tricomi equation," Journal of Mathematical Analysis and Applications, vol. 199, no. 1, pp. 271-292, 1996.

[15] K. R. Payne, "Solvability theorems for linear equations of Tricomi type," Journal of Mathematical Analysis and Applications, vol. 215, no. 1, pp. 262-273, 1997.

[16] J. Barros-Neto and I. M. Gelfand, "Fundamental solutions for the Tricomi operator," Duke Mathematical Journal, vol. 98, no. 3, pp. 465-483, 1999.

[17] C. Shuxing, "A mixed equation of Tricomi-Keldysh type," Journal of Hyperbolic Differential Equations, vol. 9, no. 3, pp. 545-553, 2012.

[18] K. Q. Zhang, "A note on initial value problem for the generalized Tricomi equation in a mixed-type domain," Acta Mathematica Sinica (English Series), vol. 29, no. 8, pp. 1581-1596, 2013.

[19] A. R. Manwell, "The Tricomi equation with applications to the theory of plane transonic flow," Recon Technical Report A 27617, 1979.

[20] P. Germain, "Remarks on the theory of partial differential equations of mixed type and applications to the study of transonic flow," Communications on Pure and Applied Mathematics, vol. 7, pp. 117-143, 1954.

[21] C. S. Morawetz, "Mixed equations and transonic flow," Journal of Hyperbolic Differential Equations, vol. 1, no. 1, pp. 1-26, 2004.

[22] S. Chen, "Mixed type equations in gas dynamics," Quarterly of Applied Mathematics, vol. 68, no. 3, pp. 487-511, 2010. 


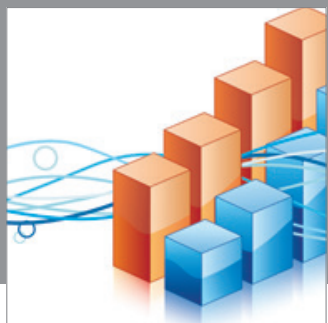

Advances in

Operations Research

mansans

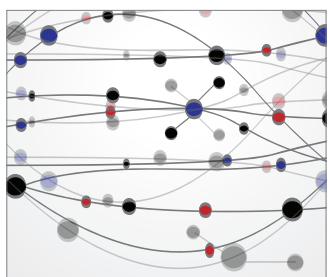

The Scientific World Journal
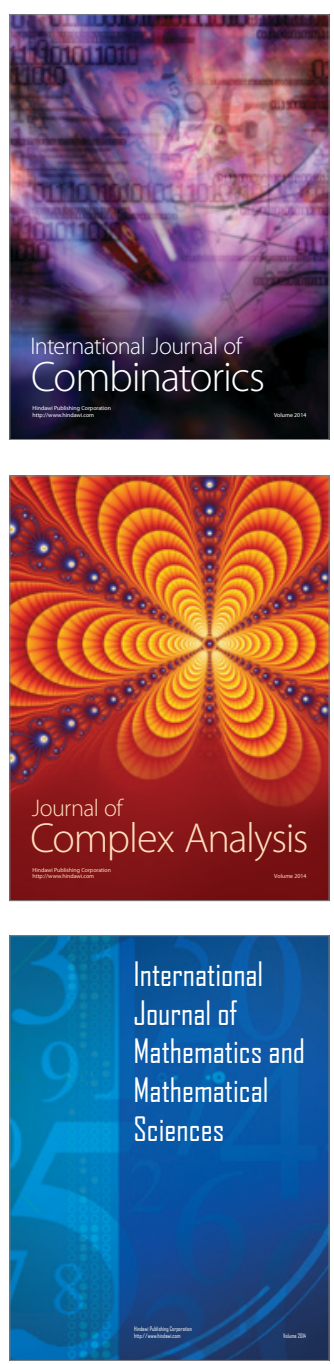
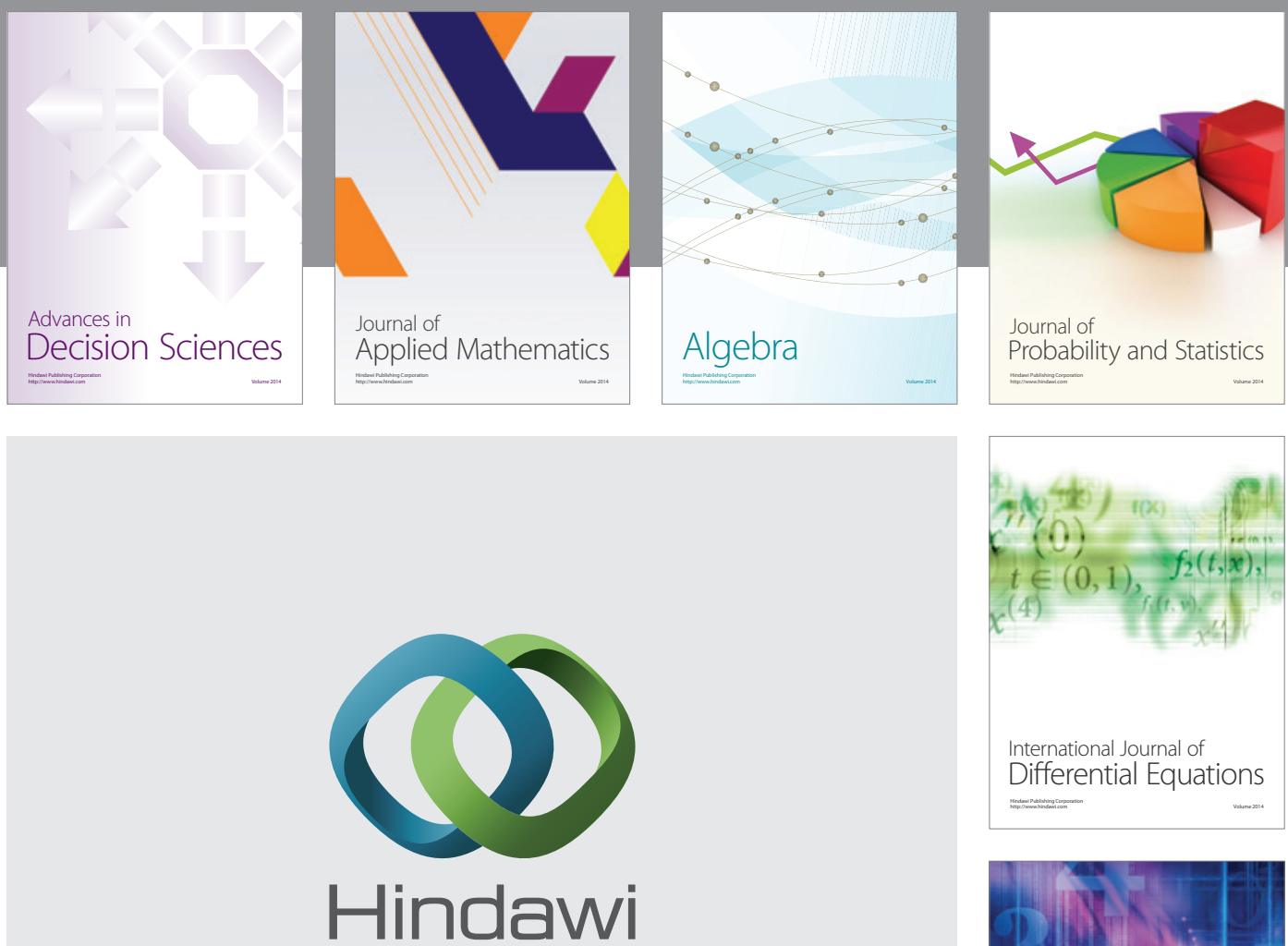

Submit your manuscripts at http://www.hindawi.com
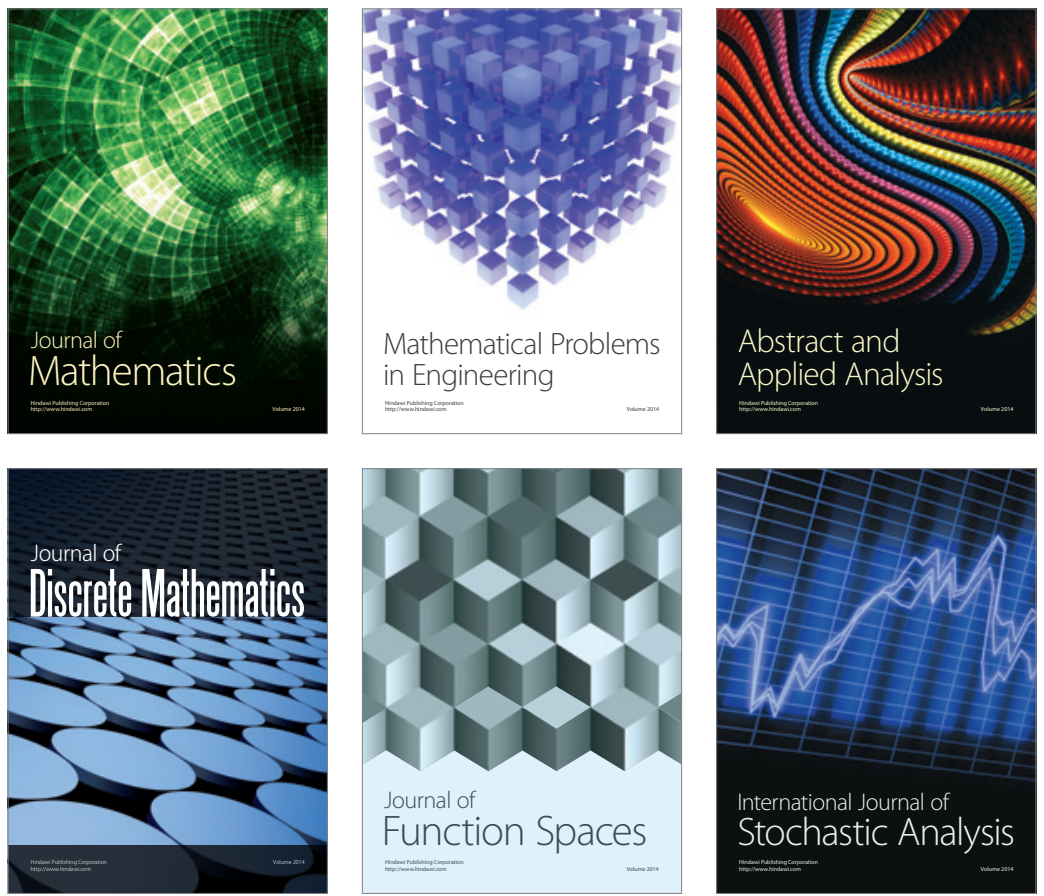

Journal of

Function Spaces

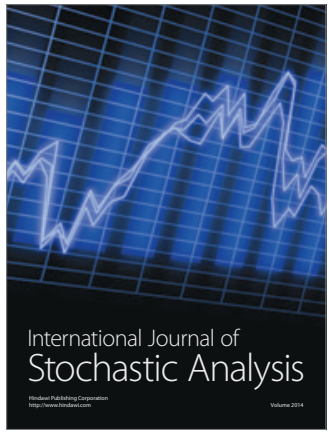

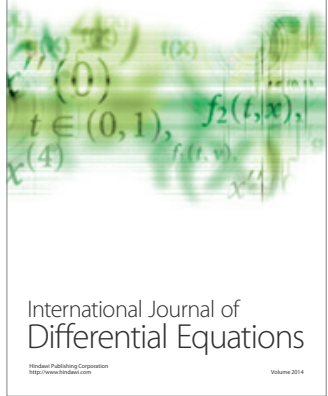
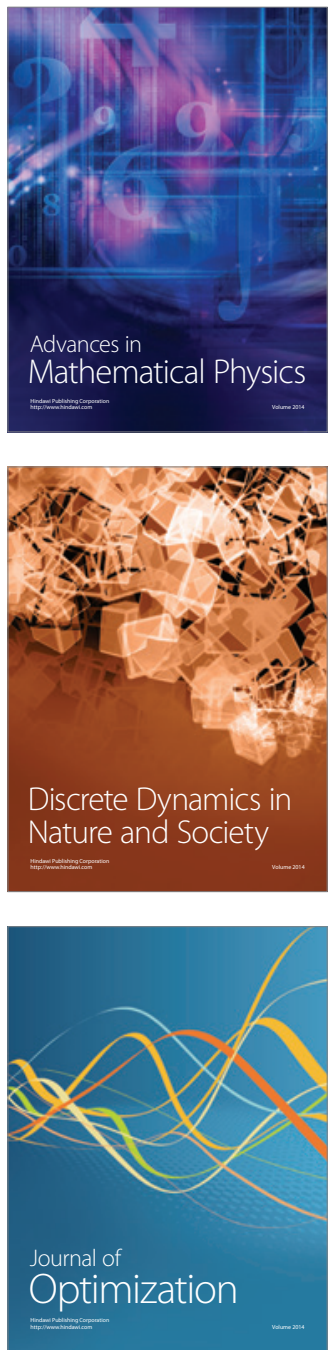